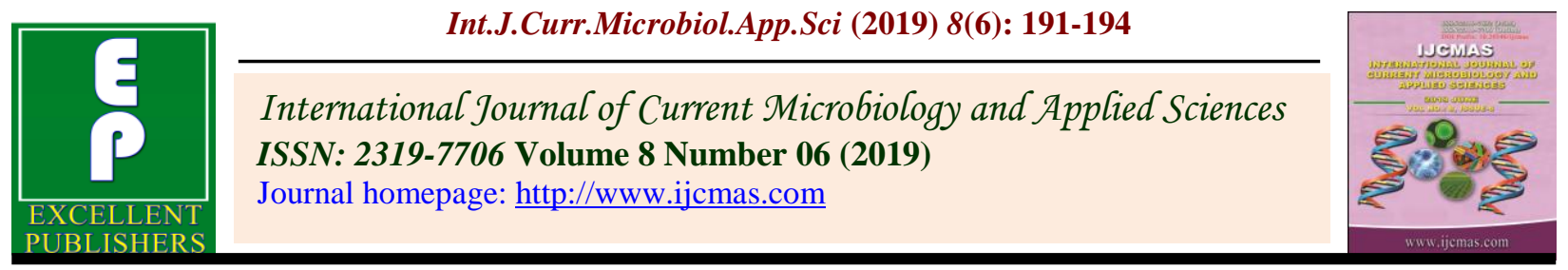

Review Article

https://doi.org/10.20546/ijcmas.2019.806.023

\title{
African Swine Fever: A Potential Threat to Pig Husbandry in India
}

\author{
T.K. Dutta*, P. Roychoudhury, Belinda L. Vangchhia, \\ Rebecca L. Ralte and P.K. Subudhi \\ Department of Veterinary Microbiology, CVSc\&AH, CAU(I), Selesih, \\ Aizawl, Mizoram - 796014, India \\ *Corresponding author
}

\begin{abstract}
A B S T R A C T
Keywords

African Swine

Fever, Pigs, India

Article Info

Accepted:

04 May 2019

Available Online:

10 June 2019

African Swine Fever (ASF) is an acute contagious vector borne disease of pigs caused by African Swine Fever virus (ASFV), which is the only known vector transmitted DNA virus and transmitted by the soft tick under genus Ornithodoros. So far the ASF was restricted to most of the African and few European countries. Recently, the disease has crossed the continental borders and reached to Asia and made a huge economic loss in Cambodia, China, Mongolia and Vietnam. It is a peracute disease of domestic and wild pigs in with mortality rate nearly $100 \%$. The disease has similarity in clinical appearance with classical swine fever, therefore, difficult to diagnose without laboratory interference. Recently, an outbreak was recorded in China bordering the village of Arunachal Pradesh. Precautionary measures need to be taken to tackle the possible threat of ASF in India, as it may cause a devastating result in the pig husbandry. The present article is prepared on information about African Swine Fever disease and possible precautionary measures to prevent the disease in India.
\end{abstract}

\section{Introduction}

African Swine Fever (ASF) is an acute contagious vector borne disease of pigs caused by African Swine Fever virus (ASFV) under genus Asfivirus and family Asfarviridae. It is the only known vector transmitted DNA virus (arbovirus). ASFV is an enveloped and icosahedral DNA virus containing a single molecule of dsDNA as genome with genome length of 170-190 kbp, which encodes about 160-.70 genes. The virus is known to be highly resistant to low temperatures. ASFV gets heat inactivated at $56^{\circ} \mathrm{C}$ for 70 minutes or at $60^{\circ} \mathrm{C}$ for 20 minutes. In addition, it is inactivated by $\mathrm{pH}$ $<3.9$ or $>11.5$ in serum-free medium. Serum increases the resistance of the virus, e.g. at $\mathrm{pH}$ 13.4 - resistance lasts up to 21 hours without serum, and 7 days with serum. The virus is susceptible to ether and chloroform. ASFV gets inactivated by $8 / 1000$ sodium hydroxide (30 minutes), hypochlorites $-2.3 \%$ chlorine (30 minutes), 3/1000 formalin (30 minutes), $3 \%$ ortho-phenylphenol (30 minutes) and iodine compounds. Alarmingly, the virus remains viable for long periods in blood, faeces and tissues; especially infected, 
uncooked or undercooked pork products. ASFV multiplies in cytoplasm of the host, as they encode all genes necessary for transcription and replication. The virus can also multiply successfully in vectors (Ornithodoros spp.).

\section{Epidemiology}

The epidemiology ofASF is complex with different epidemiological patterns of infection occurring in Africa and Europe. ASF occurs through transmission cycles involving domestic pigs, wild boars, wild African suids, and soft ticks. African wild swine (warthogs (Phacochoerus aethiopicus), bush pigs (Potamochoerus sp.), giant forest hogs (Hylochoerus meinertzhageni) are usually infected and act as reservoir hosts of ASFV in Africa. Hosts that demonstrate the disease are domestic pigs (Sus domestica), European wild boar, and American wild pigs. Ticks of the genus Ornithodoros are considered the natural arthropod host and there exists some speculation that ASFV is a virus of arthropods and that mammalian species, such as domestic swine, represent "accidental hosts".

The virus may be available in the blood, tissues, secretions and excretions of sick and dead animals infected by ASFV. Animals recovered from either acute or chronic infections may become persistently infected and can act as potential carrier of the virus, especially in African wild swine and in domestic pigs in enzootic areas. The virus used to persist for long time in soft ticks of the genus Ornithodoros.

The disease generally transmitted directly through contact between sick and healthy animals or indirectly through feeding on garbage containing infected meat (ASFV can remain infectious for 3-6 months in uncooked pork products). The most common method of transmission of the disease between wild and domestic pigs is probably through biological vectors, a soft tick, of the genus Ornithodoros. Within the tick vector transmission may occur through transstadial, transovarial as well as sexual mode. Transmission of the disease may also recorded through fomites include, premises, vehicles, implements, clothes, etc.

\section{Clinical signs}

The disease generally occurs in acute, subacute or chronic forms depending upon the virulence of the virus strain. Virulent strains cause a high mortality with an incubation period of 5-15 days; death occurs between 510 days after the onset of fever. Mortality is usually $100 \%$. In peracute cases, pigs die before appearance of gross lesions. In acute form, the affected pigs show fever $\left(40-42^{0} \mathrm{C}\right)$ of 1-2 days and gradually lose appetite and become depressed. Cyanotic patches are found over the ears, snout, belly, gluteral region, lower limbs and sometimes multiple discrete haemorrhages on the skin. A mucopurulent discharge from eyes and nose, conjunctivitis, vomiting, bleeding from nose and anus and diarrhoea are also notable clinical symptoms. In chronic form, mild signs consisting of weight loss, coughing and fluctuating temperature are seen. The course of the disease is 20-30 days or longer with low mortality. In most of the occasions, the clinical signs resemble with classical swine fever (CSF) and it is difficult to differentiate without laboratory confirmation.

Pathologically, pronounced haemorrhages in the gastrohepatic and renal lymph nodes, [etechial haemorrhages of the renal cortex and also in medulla and pelvis of kidneys are visible. Congestive splenomegaly, oedematous areas of cyanosis in hairless parts of the body, cutaneous ecchymoses on the legs and abdomen, excess of pleural, pericardial and/or peritoneal fluid and 
petechiae in the mucous membranes of the larynx and bladder, and on visceral surfaces of organs is notable pathological lesions in acute form of the disease. In chronic form, lymph nodes become enlarged and a focal caseous necrosis and mineralisation of the lungs may exist.

\section{Occurrence}

ASF is enzootic in most countries of SubSaharan Africa including Madagascar. In Europe, it has been reported and successfully eradicated from the Iberian Peninsula but continues to be found in Sardinia. In the 1970s, ASFV was present in the Caribbean (Haiti and the Dominican Republic) and one country in South America (Brazil) but was successfully eradicated. Most recently, it has appeared in the Caucasus (Georgia, Azerbaijan, and Armenia) and Russia. But in the recent past 15 countries notified ASF through immediate notifications and followup reports; 9 in Europe (Belgium, Bulgaria, Hungary, Latvia, Moldova, Poland, Romania, Russia and Ukraine); 4 in Asia (Cambodia; China (People's Republic of); Mongolia and Vietnam) and 2 in Africa (South Africa and Zimbabwe). In April, 2019 Cambodia notified the first occurrence of the disease in the country. The outbreak occurred in Rattanakiri on $22^{\text {nd }}$ March, 2019. People's Republic of China reported the first occurrence of the disease in the provinces of Xinjiang and Tibet, and the OIE also reported new outbreaks in Hubei and Yunnan. Till this report, as recorded in OIE, 97\% outbreaks of ASF in last one year occurred in Asia, where China notified the majority of all losses $(6,817$ of 7,317$)$. In February 2019, China culled over 1.01 million pigs over a period of six months, which leads to serious economic crisis as the country is the major exporter of pork and pork products worldwide. Due to the severity of the disease the World Pork Expo was cancelled, which was scheduled in April
2019. As per the OIE notifications, recent outbreak of the disease is reported from village of China nearing Arunachal Pradesh.

\section{Diagnosis}

Due to close resemblance with CSF and PRRS, diagnosis of ASF based on clinical signs, gross and microscopic lesions are rather difficult. Conclusive diagnosis is done through laboratory techniques including isolation and identification of the ASFV in primary cultures of pig monocytes or bone marrow cells. Haemadsorption test (HAD) is also commonly applicable test for definitive diagnosis of ASF, where a characteristic 'rosette' formation is detectable. ASF antigen detection by fluorescent antibody test (FAT) or detection of specific ASF viral gene by the polymerase chain reaction (PCR) techniques is particularly useful when samples may be unsuitable for virus isolation.

Serological detection of ASF antibody is not suitable in freshly affected area due to high rate of mortality. But in endemic areas indirect ELISA (the prescribed test for international trade) may be applied to detect the circulating antibody. Indirect fluorescent antibody (IFA) test should be used as a confirmatory test for sera from areas that are free from ASF and are positive in the ELISA, and for sera from endemic areas that give an inconclusive result in the ELISA. Immunoblotting test should be used as an alternative to the IFA test to confirm equivocal results with individual sera.

\section{Prevention and control}

\section{Sanitary prophylaxis}

ASFV-recovered carrier swine and persistently infected wild pigs require special consideration in controlling the disease. 


\section{Free countries}

Careful import policy for animals and animal products.

Proper disposal of waste food from aircraft or ships coming from infected countries.

Efficient sterilisation of garbage.

\section{In outbreaks}

Rapid slaughtering of all pigs and proper disposal of cadavers and litter is essential.

Thorough cleaning and disinfection.

Designation of infected zone, with control of pig movements.

Detailed epidemiological investigation, with tracing of possible sources (up-stream) and possible spread (down-stream) of infection.

Surveillance of infected zone, and surrounding area.

\section{Infected countries}

Avoid contact between pigs and soft tick vectors or their habitats (Africa) - i.e. prevent pigs from wandering.

\section{Medical prophylaxis}

No treatment

No vaccine to date

\section{Containment of ASFV}

Member Countries are reminded that the OIE Terrestrial Animal Health Code provides comprehensive guidance to Veterinary Authorities for establishing a country, zone and compartment free of African swine fever (ASF) as well as recommendations relating to the trade of pork and pork products. These products, when handled in accordance with hygienic practices complying with international standards, should not present a risk of infection. The OIE also encourages Member Countries to implement enhanced national sanitary measures on waste disposal from aircrafts/vessels/passengers and enhanced on-farm biosecurity measures including the protection of pigs from untreated swill feeding and the effective separation between domestic pigs and wild boar - and stresses the importance of OIE international standards for risk management of transboundary animal diseases (TADs) to reduce the risk of exporting disease to trading partners.

\section{References}

1. Brown C. and Torres A., Eds. (2008). USAHA Foreign Animal Diseases, Seventh Edition. Committee of Foreign and Emerging Diseases of the US Animal Health Association. Boca Publications Group, Inc.

2. Coetzer J.A.W. and Tustin R.C. Eds. (2004). - Infectious Diseases of Livestock, 2nd Edition. Oxford University Press.

3. Fauquet C., Fauquet M., and Mayo M.A. (2005). - Virus Taxonomy: VIII Report of the International Committee on Taxonomy of Viruses. Academic Press.

4. Kahn C.M., Ed. (2005). - Merck Veterinary Manual. Merck and Co. Inc. and Merial Ltd.

5. Spickler A.R., and Roth J.A. Iowa State University, College of Veterinary Medicine http://www.cfsph.iastate.edu/DiseaseInfo/fac tsheets.htm

6. World Organisation for Animal Health (2012). - Terrestrial Animal Health Code. OIE, Paris.

7. World Organisation for Animal Health (2012). - Manual of Diagnostic Tests and Vaccines for Terrestrial Animals. OIE, Paris.

\section{How to cite this article:}

Dutta, T.K., P. Roychoudhury, Belinda L. Vangchhia, Rebecca L. Ralte and Subudhi, P.K. 2019. African Swine Fever: A Potential Threat to Pig Husbandry in India. Int.J.Curr.Microbiol.App.Sci. 8(06): 191-194. doi: https://doi.org/10.20546/ijcmas.2019.806.023 